\title{
The different central nervous system efficacy among gefitinib, erlotinib and afatinib in patients with epidermal growth factor receptor mutation-positive non-small cell lung cancer
}

\author{
Hyun Ae Jung ${ }^{1}$, Sook Young Woo ${ }^{2}$, Se-Hoon Lee ${ }^{1}$, Jin Seok Ahn ${ }^{1}$, Myung-Ju Ahn ${ }^{1}$, Keunchil Park ${ }^{1}$, \\ Jong-Mu Sun ${ }^{1}$ \\ ${ }^{1}$ Division of Hematology-Oncology, Department of Medicine, Samsung Medical Center, Sungkyunkwan University School of Medicine, Seoul, \\ Korea; ${ }^{2}$ Statistics and Data Center, Samsung Medical Center, Sungkyunkwan University School of Medicine, Seoul, Korea \\ Contributions: (I) Conception and design: JM Sun, HA Jung; (II) Administrative support: HA Jung; (III) Provision of study materials or patients: HA \\ Jung, SH Lee, JS Ahn, MJ Ahn, K Park, JM Sun; (IV) Collection and assembly of data: HA Jung, SY Woo; (V) Data analysis and interpretation: JM \\ Sun, HA Jung; (VI) Manuscript writing: All authors; (VII) Final approval of manuscript: All authors. \\ Correspondence to: Jong-Mu Sun, MD, PhD. Division of Hematology-Oncology, Department of Medicine, Samsung Medical Center, Sungkyunkwan \\ University School of Medicine, 81 Irwon-ro, Gangnam-gu, Seoul 06351, Korea. Email: jongmu.sun@skku.edu.
}

Background: Brain metastasis is common in non-small cell lung cancer (NSCLC) and has an even higher incidence in epidermal growth factor receptor (EGFR)-mutant cancers. Although EGFR tyrosine kinase inhibitors (TKIs) are effective against brain metastases, it is unknown which first- or second-generation EGFR TKI is most effective.

Methods: Patients treated with first-line gefitinib, erlotinib, or afatinib for advanced EGFR-mutant NSCLC were included. The efficacy against brain metastasis was evaluated by comparing the response rates of measurable and non-irradiated brain metastases, central nervous system progression-free survival (CNSPFS), and the cumulative incidence of CNS failure.

Results: Among the 559 patients who received EGFR-TKIs (gefitinib, n=299; erlotinib, n=93; afatinib, $\mathrm{n}=167), 198$ had initial brain metastasis before starting EGFR-TKIs. The CNS response rates of gefitinib, erlotinib, and afatinib were $64.7 \%, 68.2 \%$, and $72.9 \%$, respectively $(\mathrm{P}=0.78)$. In the overall study population, irrespective of initial CNS metastasis, the median CNS-PFS was 17.3 months for gefitinib, 12.4 months for erlotinib, and 23.3 months for afatinib $(\mathrm{P}<0.001)$. In multivariate analysis for CNS-PFS, the hazard ratio (HR) of afatinib was 0.63 (95\% CI, 0.47-0.83) compared with gefitinib or erlotinib. In the competing risk analysis for cumulative incidence of CNS failure, afatinib showed a lower cumulative incidence of CNS failure compared with gefitinib or erlotinib after adjusting for both EGFR mutation type and preexisting CNS metastases (HR 0.51, 95\% CI, 0.34-0.75, P=0.0007).

Conclusions: Through there are some limitation as a retrospective study, afatinib showed similar CNS response rates, superior CNS-PFS and cumulative incidence of CNS failure, compared with gefitinib or erlotinib

Keywords: Non-small cell lung cancer (NSCLC); epidermal growth factor receptor (EGFR); tyrosine-kinase inhibitor; brain; afatinib

Submitted Mar 03, 2020. Accepted for publication Jul 20, 2020.

doi: $10.21037 /$ tlcr-20-379

View this article at: http://dx.doi.org/10.21037/tlcr-20-379 


\section{Introduction}

The treatment for non-small cell lung cancer (NSCLC) has improved over the recent decades due to new treatment modalities. However, metastasis to the central nervous system (CNS) is common in NSCLC patients and is a difficult hurdle to overcome in the treatment of NSCLC. Additionally, as magnetic resonance image (MRI) of the brain has become routine in the initial workup for NSCLC, detection of CNS metastases at initial diagnosis has increased in lung cancer patients (1). Therefore, treatment of CNS metastasis has become of paramount importance.

The incidence of baseline brain metastasis is higher in patients with epidermal growth factor receptor (EGFR)mutant NSCLC than in those with wild-type EGFR tumor $(2,3)$. In addition, the prolonged survival time afforded by EGFR tyrosine kinase inhibitors (TKIs) means that patients more frequently experience CNS metastases until they ultimately die of NSCLC. However, EGFR TKIs have higher efficacy for CNS metastases compared to cytotoxic chemotherapy in patients with EGFR-mutant NSCLC (4), and EGFR TKIs are a first-line therapy for EGFR-mutant NSCLC irrespective of baseline brain metastasis. In NCCN guideline, treatment of brain metastasis included SRS and WBRT. However, in patients with limited brain metastases and systemic therapy option with good CNS penetration, it is reasonable to hold on treating with radiation to see if systemic therapy can control the brain metastasis. Also, in the previous study, SRS followed by chemotherapy did not showed superior OS compared to chemotherapy alone in oligo-brain metastases NSCLC patients (5) .

Currently, five EGFR TKIs are available as first-line therapy for advanced EGFR-mutant NSCLC in the U.S.: gefitinib, erlotinib, afatinib, dacomitinib, and osimertinib. In a subgroup analysis of the FLAURA trial, osimertinib had better efficacy for brain metastasis than gefitinib or erlotinib $(6,7)$. However, the use of osimertinib as a first-line therapy is limited to a small population in most countries due to its high cost. Dacomitinib has not yet been approved in many countries, although it has recently been approved for use Europe. Therefore, in many countries including Korea, only three EGFR TKIs (gefitinib, erlotinib, and afatinib) are practical treatment options for patients with EGFR-mutant NSCLC.

The efficacy of gefitinib for brain metastasis in patients with EGFR-mutant NSCLC was $87.8 \%$ in one prospective study (8). A retrospective study that included NSCLC patients with brain metastasis harboring an
EGFR deletion mutation in exon 19 showed that patients treated with erlotinib had longer overall survival and progression-free survival (PFS) than patients treated with gefitinib (9). Additionally, the cerebrospinal fluid (CSF) concentration and penetration rate to the CSF from the plasma are significantly higher for erlotinib $(28.7 \mathrm{ng} / \mathrm{mL}$ and $2.77 \%$, respectively) than for gefitinib $(3.7 \mathrm{ng} / \mathrm{mL}$ and $1.13 \%$, respectively) (10). However, no studies have directly compared the CNS-specific efficacy of gefitinib and erlotinib. The CSF concentration and penetration rate to the CSF from the plasma for afatinib were $2.9 \mathrm{nM}$ and $2.5 \% \pm 2.9 \%)(11)$.

The second-generation EGFR TKI afatinib has a more potent inhibitory effect (i.e., a lower half maximal inhibitory concentration, IC50) for EGFR-mutant lung cancer cell lines than gefitinib or erlotinib (12). Although afatinib has superior efficacy for brain metastasis compared with cytotoxic chemotherapy (13), its efficacy for CNS metastasis has not been fully compared with other EGFR TKIs. In the LUX-Lung 7 trial, afatinib was directly compared with gefitinib in 319 patients with active $E G F R$-mutant NSCLC, including 51 patients with baseline brain metastases (14). Although the afatinib group had longer overall PFS than the gefitinib group, the study was too short to conclude that afatinib is better than gefitinib in the treatment of patients with brain metastasis. Additionally, the study had a small number of patients with brain metastasis, excluded those with active brain metastasis from enrollment, and had no reported efficacy information specific to brain metastasis.

In clinical practice, physicians often encounter newly diagnosed EGFR-mutant NSCLC patients with brain metastasis and need to choose an appropriate TKI as a firstline therapy. As described above, however, there is very little clinical information comparing the CNS efficacy among the three most commonly available TKIs. Therefore, this study evaluated which of the most commonly available firstor second-generation EGFR TKIs is most efficient in the control of brain metastasis and in prevention of new CNS metastasis in patients with EGFR-mutant NSCLC.

The authors present the following article in accordance with the STROBE reporting checklist (available at http:// dx.doi.org/10.21037/tlcr-20-379).

\section{Methods}

\section{Study subjects and data collection}

This study included 599 patients with EGFR-mutant 
NSCLC who started first-line gefitinib, erlotinib, afatinib treatment for advanced NSCLC at Samsung Medical Center between October 2014 and December 2016. Eligible patients were evaluated for brain metastasis using brain magnetic resonance imaging (MRI) before starting EGFR TKIs, and their medical information was extracted from electronic medical records. Patient demographic properties of age, sex, smoking history, performance status, and EGFR mutation type were reviewed. Demographic information was obtained when the first-line EGFR TKI treatment was initiated. EGFR mutations were identified using a PNAclamp ${ }^{\mathrm{TM}}$ kit and real-time polymerase chain reaction assays. Mutations other than a deletion in exon 19 or the L858R point mutation were classified as uncommon EGFR mutations.

\section{CNS response}

To evaluate CNS response, measurable brain metastatic lesions were assessed using unidimensional evaluation with Response Evaluation Criteria in Solid Tumors (RECIST) software, version 1.1 modified to allow target lesion in the brain of $5 \mathrm{~mm}$ or greater (or at least twice the slice thickness of $2.5 \mathrm{~mm}$ ) and permitting up to five target brain metastases (15). The CNS response cohort included patients who met the following criteria: (I) a measurable CNS lesion, (II) CNS lesion untreated with local therapy before starting EGFR TKIs, and (III) at least one follow-up using brain MRI during the TKI treatment. Local therapies for CNS lesions included radiation therapy, such as stereotactic radiosurgery (SRS) or whole brain radiation therapy (WBRT), and craniotomy with tumor removal. The CNS response rates were calculated as the ratio of number of patients who achieved an objective response (according to RECIST 1.1) to the total number of CNS response cohorts among the three TKI groups.

\section{Ethics statement}

This study was performed in accordance with the Declaration of Helsinki (as revised in 2013). This study was reviewed and approved by the Institutional Review Board (IRB No. 2018-02-111) at Samsung Medical Center. Consent to participate was waived by the ethics board.

\section{Statistical analysis}

The all-data cutoff date for the analyses was September 31,
2018. To compare baseline characteristics, CNS response rates, and incidence rates of new brain metastasis among the three TKIs groups, the chi-square test was used. The CNSPFS was calculated using a Kaplan-Meier estimator and compared using the log rank test. For multivariate analysis, a Cox regression model was fitted by adjusting the prognostic variables deemed significant $(\mathrm{P}<0.05)$ in univariate analysis of PFS. CNS-PFS is presented as the median value with two-sided 95\% CI. CNS-PFS was defined as the first date of EGFR-TKIs until CNS progression [i.e., new brain or leptomeningeal metastasis or increase of preexisting CNS lesions, according to RECIST 1.1 (16)] or death resulting from any cause. Patients with no CNS progression or death at the time of the analysis were censored as of the last day of administration of EGFR-TKIs.

A competing risk analysis estimating the cumulative incidence for the event of interest (CNS progression) in the presence of two competing risk events (nonCNS progression and death) was performed using a semiparametric Fine-Gray regression model. Event time was defined as either occurrence of the earliest of the three events or patients were censored at the time of their last assessment. Statistical analysis was executed using $\mathrm{R}$ software, version 3.5.1, with the cmprsk package (Vienna, Austria; http://www.R-project.org). Cumulative incidence was adjusted for EGFR mutation type and preexisting CNS metastases before EGFR TKI treatment. P-value and 95\% CI, for HR were corrected using Bonferroni's method due to multiple testing with a raw $\mathrm{P}$-value multiplied by 2 in subgroup analysis (one group with preexisting CNS metastases and one group without). All P-values were twosided, and $\mathrm{P}<0.05$ was considered statistically significant.

\section{Results}

\section{Patient characteristics}

The total number of patients treated with first-line EGFRTKIs during the study period was 559. Table 1 summarizes the baseline characteristics of these patients. During the study period, 299, 93, and 167 patients started first-line gefitinib, erlotinib, and afatinib treatment, respectively. Compared to the other two groups, the afatinib group was younger and had more mutations involving deletions in exon 19, the gefitinib group had more female patients and patients who had never smoked, and the erlotinib group had more patients with initial brain metastasis before starting EGFR TKI. There was no difference in CNS tumor 
Table 1 Baseline characteristics

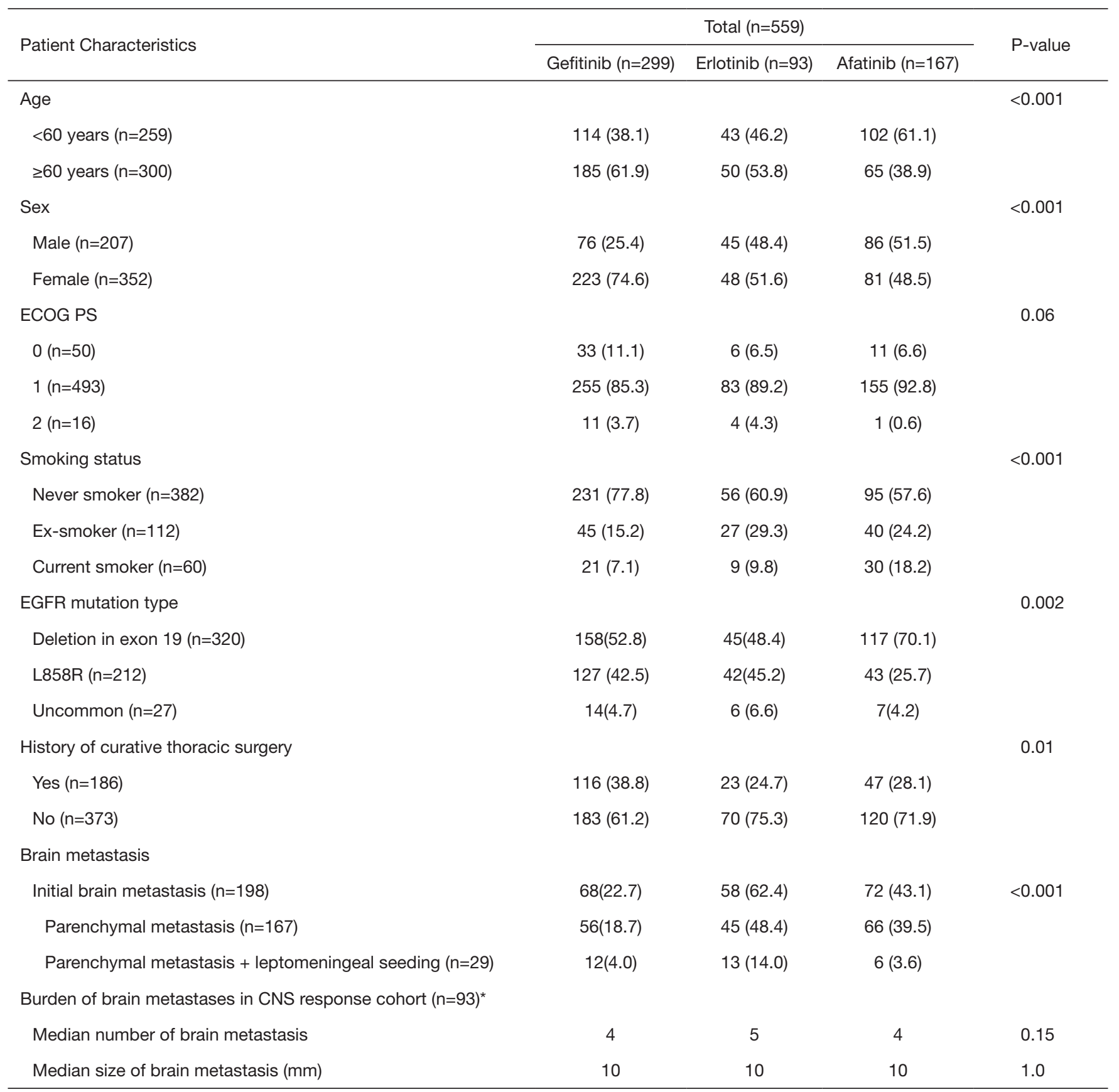

Values are presented as $\mathrm{n}(\%)$. Uncommon EGFR was defined as tumor containing mutation other than del19 or L858R. *, Gefitinib ( $\mathrm{n}=24)$, Erlotinib ( $n=22)$, Afatinib ( $n=37$ ). ECOG, Eastern Cooperative Oncology Group; PS, performance status; EGFR, epidermal growth factor receptor. 
Table 2 Treatment patterns and outcomes of brain metastasis

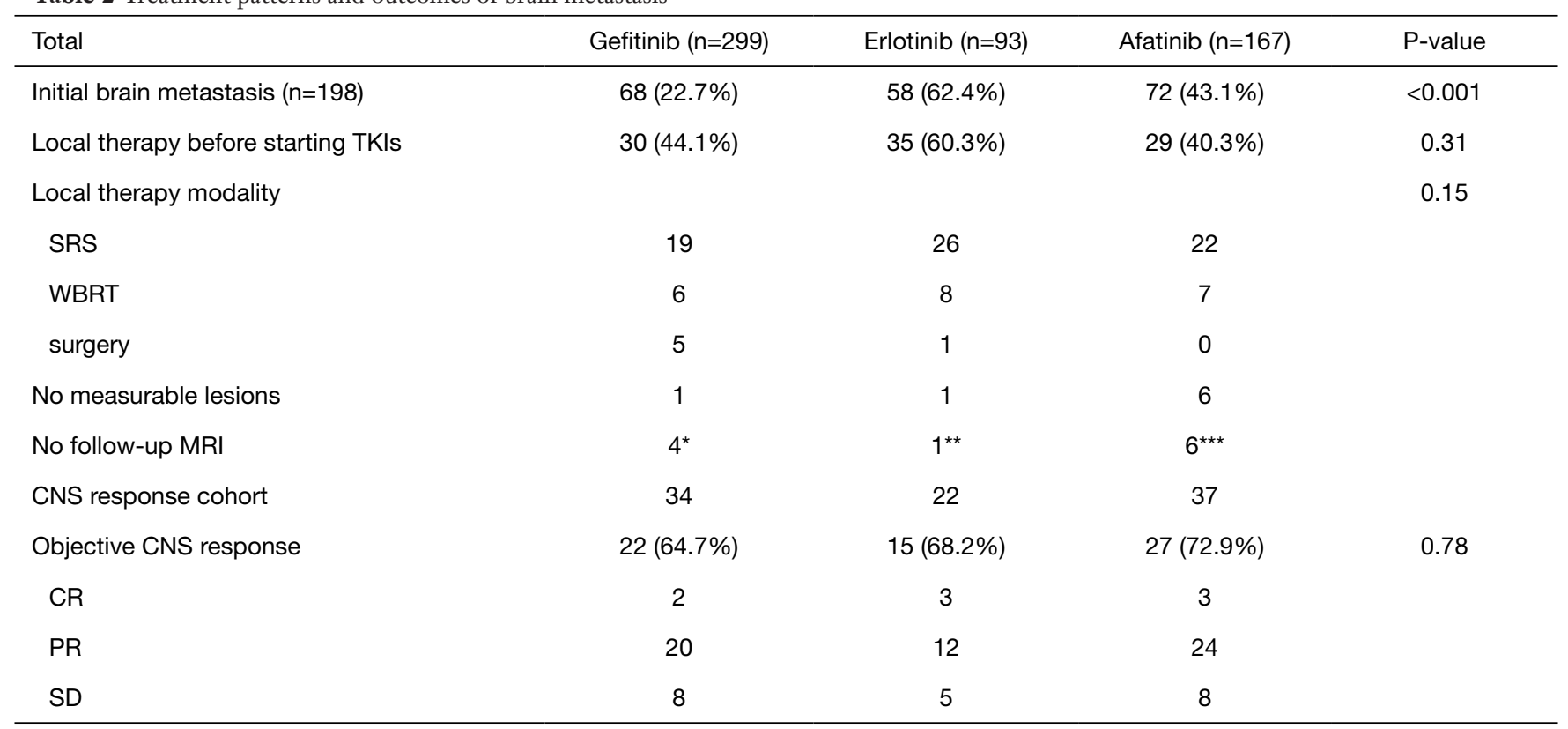

All values are presented as either $n(\%)$ or $n .{ }^{*}$, of the four patients, one also had no measurable lesion; ${ }^{\star *}$, the patient had no measurable lesion; ${ }^{* *}$, all six patients had no measurable lesion. TKI, tyrosine-kinase inhibitor; SRS, stereotactic radiosurgery; WBRT, whole-brain radiotherapy; CNS, central nervous system; CR, complete response; PR, partial response; SD, stable disease.

burden among three groups, in terms of number or size of brain metastasis in the CNS response cohort. The median treatment duration was $14.9,11.2$, and 17.7 months for gefitinib, erlotinib, and afatinib, respectively.

\section{Overall survival for the three EGFR-TKIs}

Median OS were 33.0, 31.1 and 33.1 months for gefitinib, erlotinib and afatinib respectively. There was no statistically significant difference of median OS among the three EGFR-TKIs ( $\mathrm{P}=0.98)$ (Figure S1).

\section{CNS response rates}

Out of the 559 study participants, 198 (35.4\%) had initial brain metastases, 94 of whom received local therapy, specifically SRS $(n=67)$, WBRT $(n=21)$, or surgery $(n=6)$, before starting EGFR TKIs. Of the 104 patients who did not receive local therapy for CNS lesions, 11 had no measurable lesion or follow-up brain MRI for response evaluation. Therefore, the remaining 93 patients were included in the CNS-response cohort, and the objective CNS response rate was $64.7 \%(n=22 / 34)$ for gefitinib, $68.2 \%(n=15 / 22)$ for erlotinib, and $72.9 \%(n=27 / 37)$ for afatinib $(\mathrm{P}=0.78)$ (Table 2). Table $S 1$ showed the number of brain metastases and maximum size in CNS response cohort.

\section{CNS progression-free survival}

We evaluated CNS-PFS for the entire study population ( $\mathrm{n}=599)$ with or without preexisting CNS metastases. CNS progression occurred in a total of 157 patients at a rate of $30.8 \%$ (92/299), 31.2\% (29/93), and 21.6\% (36/167) in the gefitinib, erlotinib, and afatinib groups, respectively (Figure 1A). The median CNS-PFS was 17.3 months (range, 13.7-20.8 months), 12.4 months (range, 9.3-15.5 months), and 23.3 months (range, 16.4-30.2 months) for the gefitinib, erlotinib, and afatinib groups, respectively $(\mathrm{P}<0.001)$. In the univariate analysis, afatinib treatment, deletions in exon 19 , and absence of initial CNS metastases were significantly associated with longer CNS-PFS, and those associations held in the multivariate analysis (Table 3).

\section{Cumulative incidence of CNS failure in the entire study population}

Figure $1 B$ shows the cumulative incidences of CNS failure 

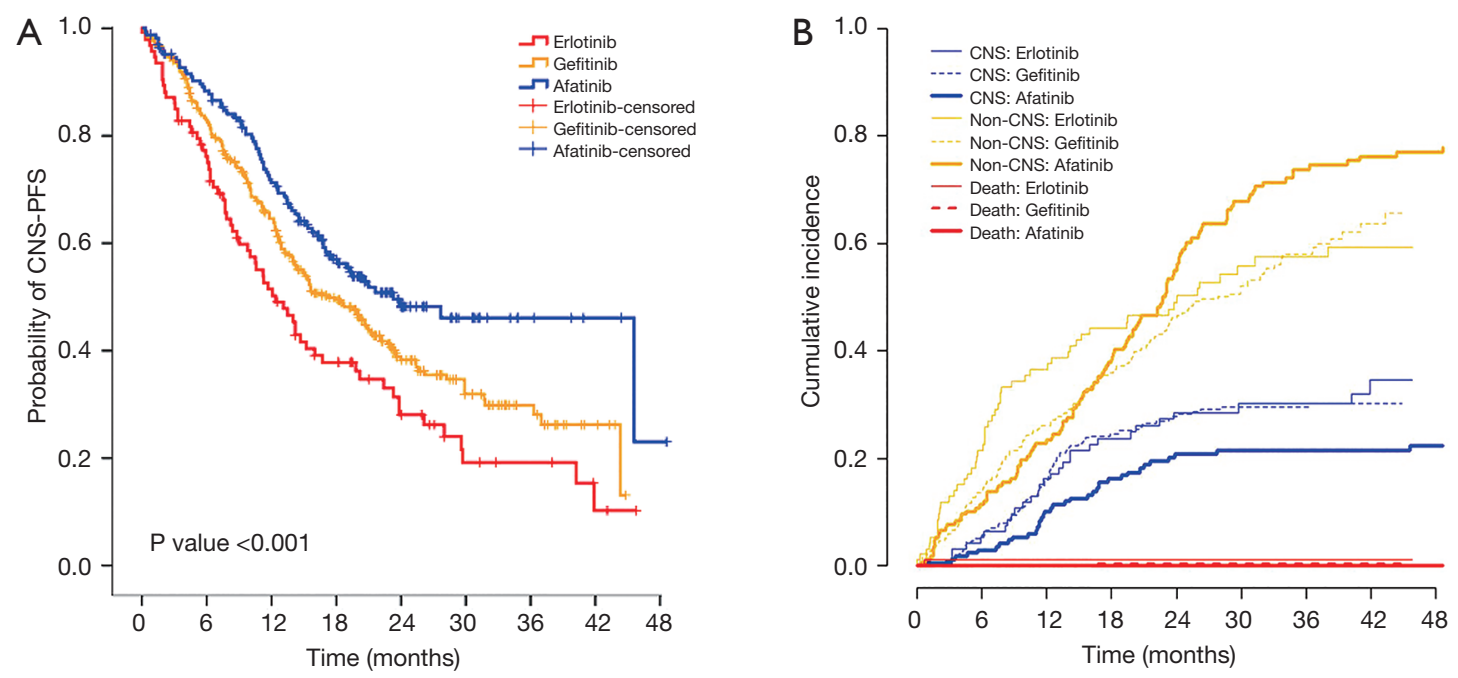

Figure 1 Afatinib had a superior tendency in terms of CNS-PFS, and cumulative incidence of CNS failure compared with gefitinib or erlotinib in patients in study populations. (A) CNS-PFS in the three TKIs in all study populations; (B) cumulative incidence of CNS failure in all study populations. CNS-PFS, central nervous system progression-free survival; TKI, tyrosine kinase inhibitor.

Table 3 Analysis for CNS-PFS

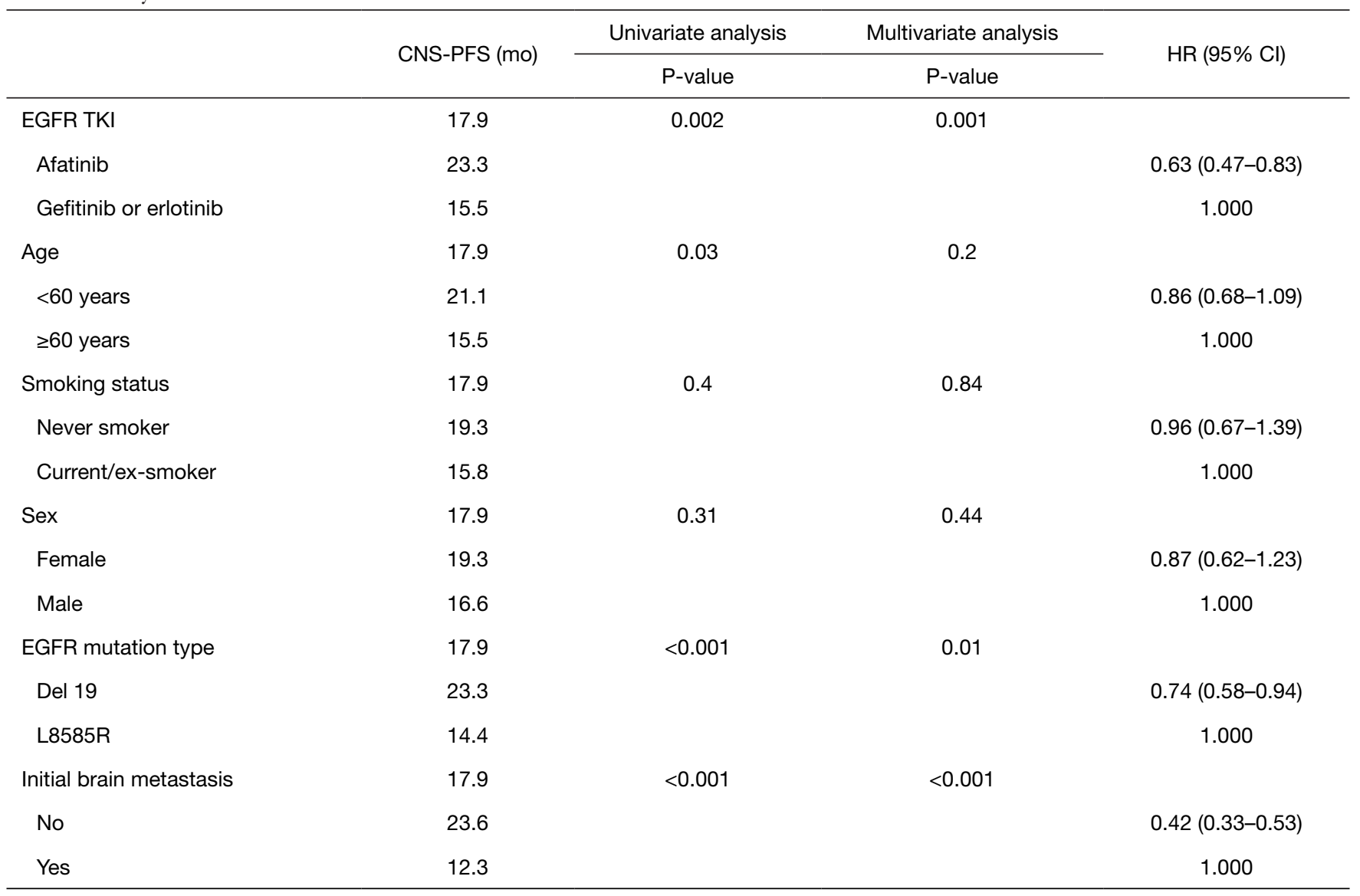

CNS-PFS, central nervous system progression-free survival; EGFR, epidermal growth factor receptor; TKI, tyrosine kinase inhibitor. 
Table 4 Cumulative incidence of CNS failure

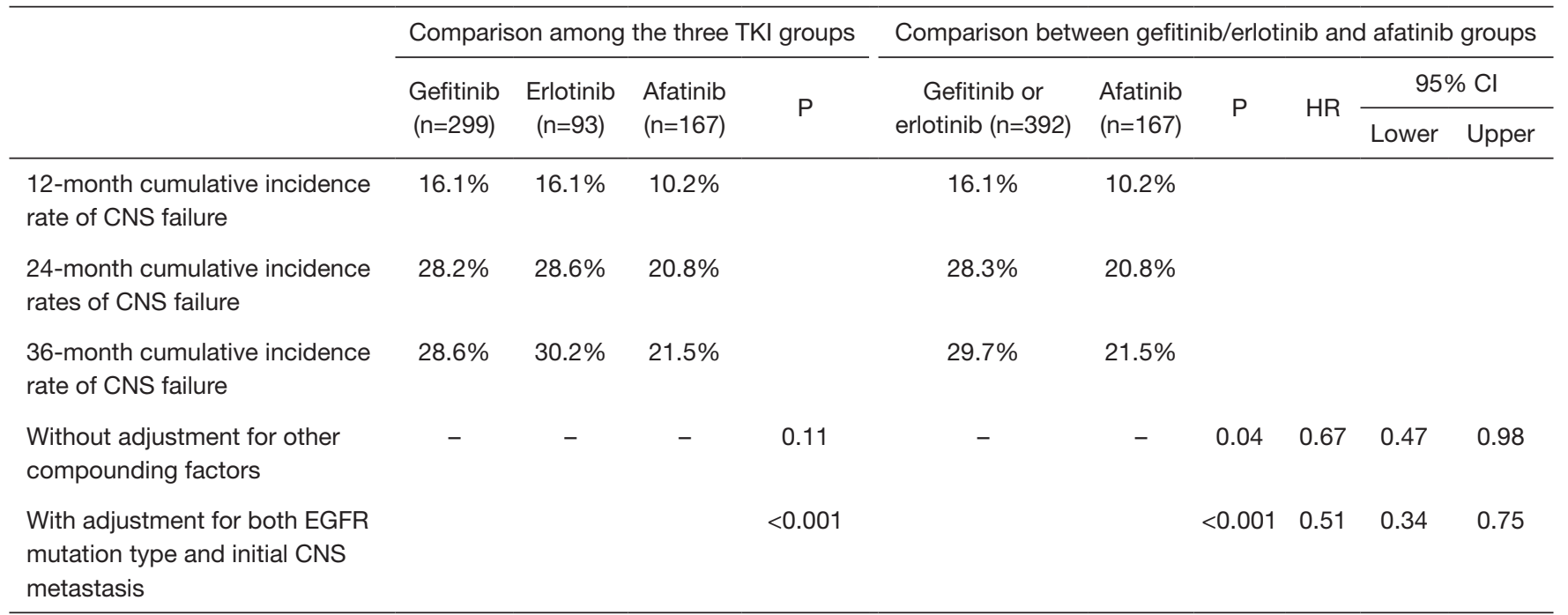

CNS, central nervous system; TKI, tyrosine-kinase inhibitor.

in the entire study population according to TKI type. The competing risk analysis estimated the probability of CNS progression at 12 months as $16.1 \%, 16.1 \%$, and $10.2 \%$ for gefitinib, erlotinib, and afatinib, respectively. At 24 months, the probabilities were $28.2 \%, 28.6 \%$, and $20.8 \%$ for gefitinib, erlotinib, and afatinib, respectively $(\mathrm{P}=0.11)$ (Table 4).

After adjusting for possible compounding factors, such as EGFR mutation type and preexisting CNS metastasis, the cumulative incidences of CNS failure were significantly different among the three EGFR TKI groups $(\mathrm{P}<0.001)$ (Table 4, Figure 1B). When afatinib was compared with gefitinib and erlotinib (as a compound group), it significantly reduced the cumulative incidence of CNS failure (HR 0.51, 95\% CI, 0.34-0.75, $\mathrm{P}=0.0007$ ) (Table 4).

\section{Cumulative incidence of CNS failure according to initial existence of brain metastases}

Since the existence of initial brain metastasis may affect the CNS efficacy of TKIs, we performed subgroup analysis on the cumulative incidence of CNS failure according to initial brain metastasis. Of the 198 patients with initial CNS metastases, 88 showed a CNS failure event. The gefitinib group had a significantly higher cumulative incidence of CNS failure compared with the erlotinib or afatinib groups, with 24 -month cumulative rates of $59.3 \%, 28.1 \%$, and $35.4 \%$, respectively $(\mathrm{P}<0.001)$ (Figure $2 A)$. After adjusting for EGFR mutation type and history of local therapy before starting TKI therapy, the difference was still significant $(\mathrm{P}=0.004)$.

Of the 361 patients without initial CNS metastases, 69 developed new CNS metastases during EGFR TKI treatment. The total incidence rate of new CNS metastasis in this subgroup was $21.2 \%$ (49/231) for gefitinib, $28.6 \%$ $(10 / 35)$ for erlotinib, and $11.8 \%(10 / 95)$ for afatinib $(\mathrm{P}=0.04)$. The 24-month cumulative incidence rate of new CNS metastasis was $9.6 \%$ for afatinib, which was lower than those of gefitinib (18.5\%) and erlotinib (29.3\%) ( $\mathrm{P}=0.09)$ (Figure 2B). After adjusting for EGFR mutation type, the $\mathrm{P}$-value decreased to 0.07 .

\section{Discussion}

Our study shows afatinib to have superior CNS efficacy (longer CNS-PFS and lower cumulative incidence of CNS failure) compared with gefitinib or erlotinib in patients with $E G F R$-mutant NSCLC. Our previous study with a similar population also found that the afatinib group had longer overall PFS than the gefitinib or erlotinib groups (17). Together with the present data, the longer PFS of afatinib may be in part attributed to its superior CNS efficacy compared with gefitinib or erlotinib.

We used two statistical methods for the time-to-event analysis for CNS efficacy: CNS-PFS and cumulative incidence of CNS failure. In the analysis of cumulative incidence of CNS failure, patients who experienced non- 

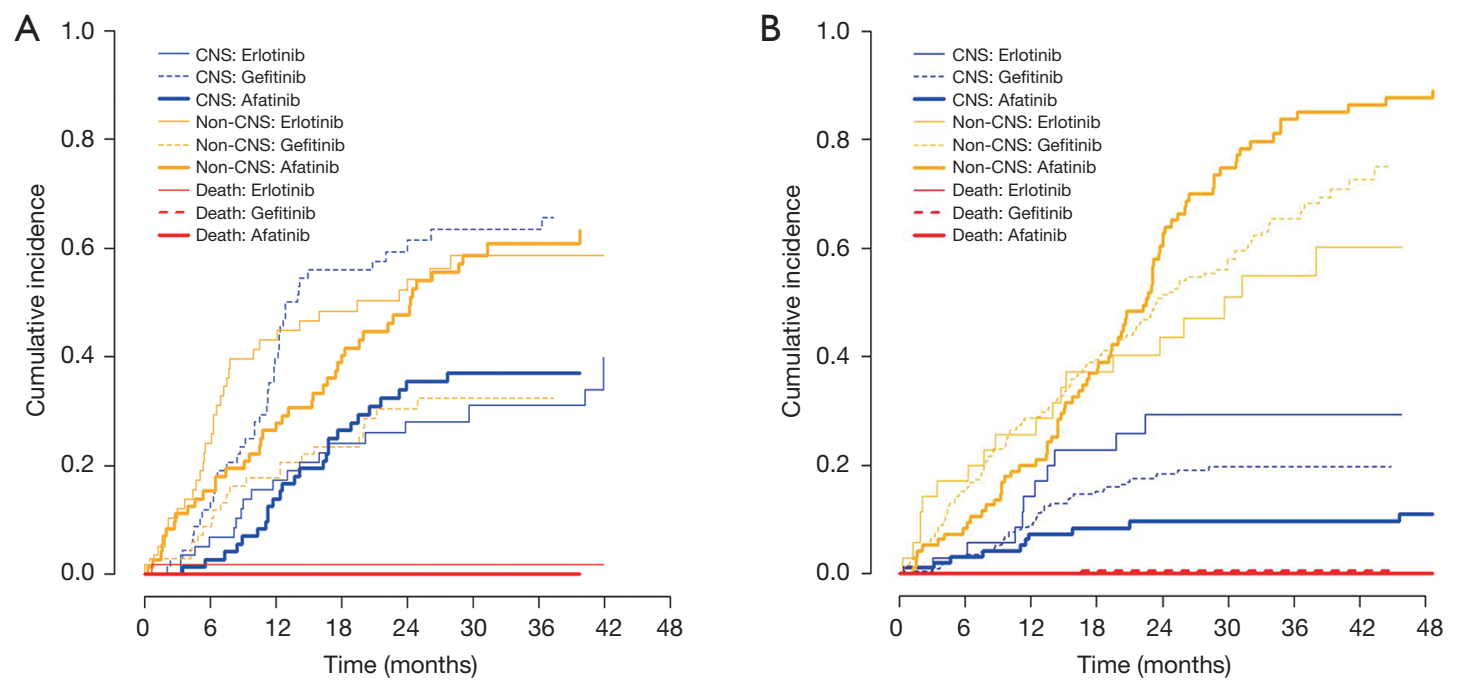

Figure 2 Afatinib had a superior tendency in terms of CNS-PFS, and cumulative incidence of CNS failure compared with gefitinib or erlotinib in patients with initial brain metastasis. (A) Cumulative incidence of CNS failure in patients with initial brain metastasis; (B) Cumulative incidence of CNS failure in patients without initial brain metastasis. CNS, central nervous system.

CNS progression or death before CNS progression were censored to exclude the confounding effects of the two competing events (18). After this adjustment, we found a different pattern of cumulative incidence of CNS failure (Figure $1 B$ ) from that of CNS-PFS (Figure 1A) in that the curves for the two first-generation TKIs were similar to each other but slightly separated from the curve for afatinib (19).

In addition, several reports have shown that brain metastasis in patients with tumors harboring a deletion in exon 19 had distinguishing radiographic features and favorable outcomes compared with other EGFR mutations including L858R $(8,20)$. Our study also found that a deletion in exon 19, as well was the initial existence of brain metastasis, was associated with longer PFS. After adjusting for these prognostic factors, the protective effect of afatinib became more significant compared with that of gefitinib or erlotinib (HR 0.51, 95\% CI, 0.34-0.75) (Table 4).

There were significant differences in the proportion of patients with initial brain metastasis, among the gefitinib (22.7\%), erlotinib (62.4\%), and afatinib (43.1\%) groups. Since the choice of TKI was performed depending on physician discretion in this study, the TKI treatment chosen for patients with brain metastasis may have been affected by physician awareness of previous studies favoring erlotinib or afatinib in terms of blood-to-brain penetration rates or IC50 level (10-12).

For the cumulative incidence of CNS failure, erlotinib showed comparable outcomes to afatinib in the subgroup with initial brain metastasis but worse outcomes in the subgroup with no initial brain metastasis (Figure 2). Although the reason for this discrepancy is unclear, the larger proportion of patients treated with local therapy before starting TKIs in the erlotinib group than in the afatinib group ( $60 \%$ vs. $40 \%)$ may have affected the assessment of CNS efficacy of TKI therapy in the subgroup with initial brain metastasis.

The non-neglectable incidence rates of CNS failure in the subgroup without initial brain metastasis indicate that the CNS efficacy of EGFR TKIs should be considered for all patients regardless of initial brain metastasis. The 24-month-cumulative incidence rate of new brain metastasis in the gefitinib and erlotinib groups was approximately two (18.5\%) and three times (29.3\%), respectively, that of the afatinib group $(9.6 \%)$. Since other biases, such as prior local therapy, could not have affected this analysis of CNS efficacy, these data more accurately represent the superior efficacy of afatinib.

Our study has significant limitation by its retrospective nature. We had not assessed CNS status or checked brain images in a preplanned manner. As a result, the most important bias is the irregular follow-up schedule of brain MRI among the study population. All patients included in this study were evaluated using brain MRI as part of their staging workup before starting first-line EGFR TKI 
therapy. However, 31.1\% (174/559) of patients who did not complained of CNS symptoms did not receive followup brain MRI during EGFR TKI therapy. In addition, follow-up brain MRI was performed based either on patient reported symptoms, which were possibly associated with CNS progression, or on an individual physician's tendency to check for asymptomatic CNS progression and not based on a pre-planned schedule. However, it can be reasonably assumed that the follow-up MRI criteria were universally applied in all three TKI groups so that any bias would not significantly affect the comparative analysis among the three TKI groups. The second limitation is the possibility of a different burden of brain metastasis among the three TKI groups. To overcome this limitation, we performed subgroup analysis according to the existence of initial brain metastasis. However, the burden can vary even within a subgroup with initial brain metastasis. The high proportion of local therapy, including WBRT, in the erlotinib group suggests a slightly larger burden in that group. The choice of selection of the EGFR-TKIs was by individual physician. There were major imbalances among the three TKI groups, especially exon 19 mutations were present in $70 \%$ of the afatinib group compared to $50 \%$ in the other two groups. Also, we had very limited clinical data on the CNS efficacy of the newly approved second-generation TKI dacomitinib because the phase III study comparing it with gefitinib did not include any patients with brain metastasis (21). Based on a previous mouse study showing its high blood-tobrain penetration rate (22), dacomitinib is expected to have excellent CNS efficacy comparable with afatinib, although it must be further investigated clinically.

Although these limitations exist, our study is the largest comparing CNS efficacy between first- or secondgeneration EGFR TKIs and shows the superiority of second-generation TKIs. Because a prospective study comparing CNS efficacy between first- or secondgeneration TKIs is not likely to be conducted in the near future, our study provides guidance for clinical practice.

\section{Conclusions}

In this study, we evaluated patterns of treatment and their outcomes for brain metastasis in patients with EGFRmutant NSCLC. Our study showed that the CNS response rates were similar among three EGFR-TKIs. Afatinib had a superior tendency in terms of CNS-PFS, and cumulative incidence of CNS failure compared with gefitinib or erlotinib. However, our study, as a retrospective study, has significant limitation for the accurate comparison of CNS efficacy, so cautious interpretation or prospective studies are necessary before the clinical application of our data.

\section{Acknowledgments}

Funding: None.

\section{Footnote}

Reporting Checklist: The authors have completed the STROBE reporting checklist. Available at http://dx.doi. org/10.21037/tlcr-20-379

Data Sharing Statement: Available at http://dx.doi. org/10.21037/tlcr-20-379

Conflicts of Interest: All authors have completed the ICMJE uniform disclosure form (available at http://dx.doi. org/10.21037/tlcr-20-379). The authors have no conflicts of interest to declare.

Etbical Statement: The authors are accountable for all aspects of the work in ensuring that questions related to the accuracy or integrity of any part of the work are appropriately investigated and resolved. This study was performed in accordance with the Declaration of Helsinki (as revised in 2013). This study was reviewed and approved by the Institutional Review Board (IRB No. 2018-02-111) at Samsung Medical Center. Consent to participate was waived by the ethics board.

Open Access Statement: This is an Open Access article distributed in accordance with the Creative Commons Attribution-NonCommercial-NoDerivs 4.0 International License (CC BY-NC-ND 4.0), which permits the noncommercial replication and distribution of the article with the strict proviso that no changes or edits are made and the original work is properly cited (including links to both the formal publication through the relevant DOI and the license). See: https://creativecommons.org/licenses/by-nc-nd/4.0/.

\section{References}

1. Mayr NA, Hussey DH, Yuh W'T. Cost-effectiveness of high-contrast-dose MR screening of asymptomatic brain metastasis. AJNR Am J Neuroradiol 1995;16:215-7.

2. Iuchi T, Shingyoji M, Itakura $M$, et al. Frequency of 
brain metastases in non-small-cell lung cancer, and their association with epidermal growth factor receptor mutations. Int J Clin Oncol 2015;20:674-9.

3. Ge M, Zhuang Y, Zhou X, et al. High probability and frequency of EGFR mutations in non-small cell lung cancer with brain metastases. J Neurooncol 2017;135:413-8.

4. Watanabe $\mathrm{S}$, Hayashi $\mathrm{H}$, Nakagawa K. Is afatinib a treatment option for brain metastases in patients with EGFR mutation-positive non-small cell lung cancer? Ann Transl Med 2016;4:225.

5. Lim SH, Lee JY, Lee MY, et al. A randomized phase III trial of stereotactic radiosurgery (SRS) versus observation for patients with asymptomatic cerebral oligo-metastases in non-small-cell lung cancer. Ann Oncol 2015;26:762-8.

6. Wu YL, Ahn MJ, Garassino MC, et al. CNS Efficacy of Osimertinib in Patients With T790M-Positive Advanced Non-Small-Cell Lung Cancer: Data From a Randomized Phase III Trial (AURA3). J Clin Oncol 2018;36:2702-9.

7. Soria JC, Ohe Y, Vansteenkiste J, et al. Osimertinib in Untreated EGFR-Mutated Advanced Non-Small-Cell Lung Cancer. N Engl J Med 2018;378:113-25.

8. Iuchi T, Shingyoji M, Sakaida T, et al. Phase II trial of gefitinib alone without radiation therapy for Japanese patients with brain metastases from EGFR-mutant lung adenocarcinoma. Lung Cancer 2013;82:282-7.

9. Jiang Y, Zhang J, Huang J, et al. Erlotinib versus gefitinib for brain metastases in Asian patients with exon 19 EGFRmutant lung adenocarcinoma: a retrospective, multicenter study. BMC Pulm Med 2018;18:171.

10. Togashi Y, Masago K, Masuda S, et al. Cerebrospinal fluid concentration of gefitinib and erlotinib in patients with non-small cell lung cancer. Cancer Chemother Pharmacol 2012;70:399-405.

11. Tamiya A, Tamiya M, Nishihara T, et al. Cerebrospinal Fluid Penetration Rate and Efficacy of Afatinib in Patients with EGFR Mutation-positive Non-small Cell Lung Cancer with Leptomeningeal Carcinomatosis: A Multicenter Prospective Study. Anticancer Res 2017;37:4177-82.

12. Li D, Ambrogio L, Shimamura T, et al. BIBW2992, an irreversible EGFR/HER2 inhibitor highly effective in preclinical lung cancer models. Oncogene 2008;27:4702-11.

13. Schuler M, Wu YL, Hirsh V, et al. First-Line Afatinib versus Chemotherapy in Patients with Non-Small Cell Lung Cancer and Common Epidermal Growth Factor
Receptor Gene Mutations and Brain Metastases. J Thorac Oncol 2016;11:380-90.

14. Park K, Tan EH, O'Byrne K, et al. Afatinib versus gefitinib as first-line treatment of patients with EGFR mutationpositive non-small-cell lung cancer (LUX-Lung 7): a phase 2B, open-label, randomised controlled trial. Lancet Oncol 2016;17:577-89.

15. Qian JM, Mahajan A, Yu JB, et al. Comparing available criteria for measuring brain metastasis response to immunotherapy. J Neurooncol 2017;132:479-85.

16. Eisenhauer EA, Therasse P, Bogaerts J, et al. New response evaluation criteria in solid tumours: revised RECIST guideline (version 1.1). Eur J Cancer 2009;45:228-47.

17. Kim Y, Lee SH, Ahn JS, et al. Efficacy and Safety of Afatinib for EGFR-mutant Non-small Cell Lung Cancer, Compared with Gefitinib or Erlotinib. Cancer Res Treat 2019;51:502-9.

18. Kim HT. Cumulative incidence in competing risks data and competing risks regression analysis. Clin Cancer Res 2007;13:559-65.

19. Varadhan R, Weiss CO, Segal JB, et al. Evaluating health outcomes in the presence of competing risks: a review of statistical methods and clinical applications. Med Care 2010;48:S96-105.

20. Sekine A, Kato T, Hagiwara E, et al. Metastatic brain tumors from non-small cell lung cancer with EGFR mutations: distinguishing influence of exon 19 deletion on radiographic features. Lung Cancer 2012;77:64-9.

21. Wu YL, Cheng Y, Zhou X, et al. Dacomitinib versus gefitinib as first-line treatment for patients with EGFRmutation-positive non-small-cell lung cancer (ARCHER 1050): a randomised, open-label, phase 3 trial. Lancet Oncol 2017;18:1454-66.

22. Kim M, Laramy JK, Mohammad AS, et al. Brain Distribution of a Panel of Epidermal Growth Factor Receptor Inhibitors Using Cassette Dosing in Wild-Type and Abcb1/Abcg2-Deficient Mice. Drug Metab Dispos 2019;47:393-404.

Cite this article as: Jung HA, Woo SY, Lee SH, Ahn JS, Ahn MJ, Park K, Sun KM. The different central nervous system efficacy among gefitinib, erlotinib and afatinib in patients with epidermal growth factor receptor mutation-positive non-small cell lung cancer. Transl Lung Cancer Res 2020;9(5):1749-1758. doi: $10.21037 /$ tlcr-20-379 
Supplementary

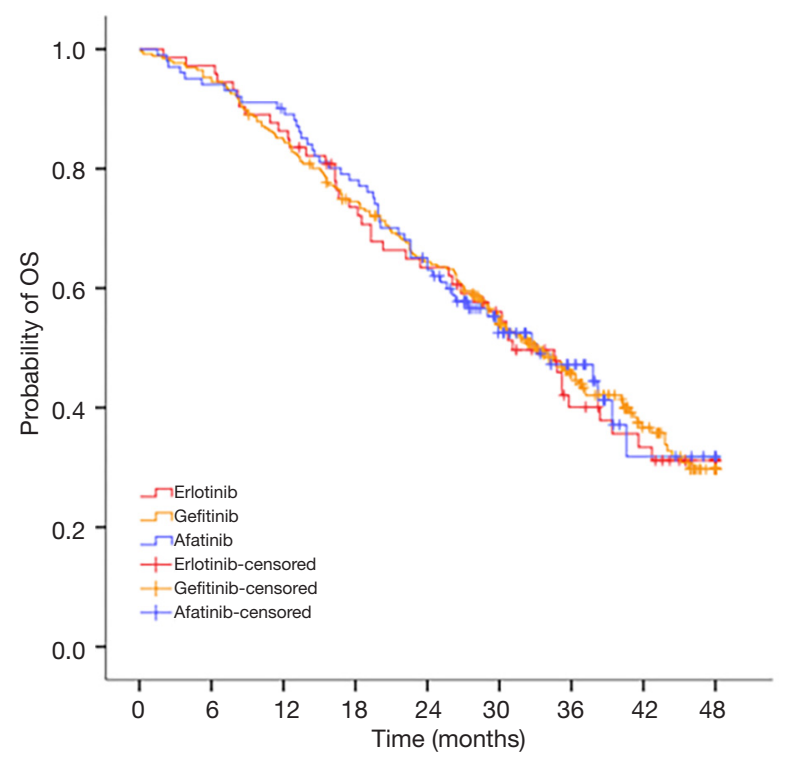

Figure S1 OS in all study population.

Table S1 The number of brain metastases and maximum size in CNS response cohort

\begin{tabular}{lccccc}
\hline & Brain metastases & Gefitinib $(\mathrm{n}=34)$ & Erlotinib $(\mathrm{n}=22)$ & Afatinib $(\mathrm{n}=37)$ & P value \\
\hline Number & Single & 14 & 3 & 10 & 0.074 \\
$2-5$ & 13 & 9 & 19 & \\
\multicolumn{1}{l}{ Maximum size [median: min-max] } & 7 & 10 & 8 & \\
\hline
\end{tabular}

CNS, central nervous system. 